\title{
Dietary immunostimulants influence specific immune response and resistance of healthy and immunocompromised Asian catfish Clarias batrachus to Aeromonas hydrophila infection
}

\author{
Jaya Kumari, P. K. Sahoo* \\ Central Institute of Freshwater Aquaculture, Kausalyaganga, Bhubaneswar 751 002, India
}

\begin{abstract}
In order to determine the efficacy and immunoreversal effect of the 4 dietary immunomodulators, viz. lactoferrin, $\beta-1,3$ glucan, levamisole and vitamin $C$, on disease resistance of a commercially important catfish, Clarias batrachus, fish were fed diets supplemented with various levels of these substances in 2 subgroups, healthy and immunocompromised, during a $30 \mathrm{~d}$ trial. An artificial immunosuppressive state was induced by giving 3 intraperitoneal (i.p.) injections of cyclophosphamide (CYP) at a dose level of $200 \mathrm{mg} \mathrm{kg}^{-1}$ body weight at 1 wk intervals in the immunocompromised vaccinated subgroup and 3 consecutive injections $3 \mathrm{~d}$ before challenge in the immunocompromised non-vaccinated subgroup. On the first day of the experiment, the fish were vaccinated against a formalin-killed Aeromonas hydrophila bacterin. After $30 \mathrm{~d}$, antibody titre (as measured through bacterial agglutination titre) and disease resistance against $A$. hydrophila were determined. The results demonstrate that all 4 immunomodulators were capable of significantly $(p<0.05)$ enhancing the specific immune response; this was evident through raised antibody titre and protection against $A$. hydrophila in both healthy and immunocompromised vaccinated subgroups compared to their respective controls. Similarly, all 4 substances significantly raised the survival rates in immunocompromised and healthy non-vaccinated fish. Thus, these substances were capable of reducing the immunosuppression induced by CYP injections in both vaccinated and non-vaccinated fish compared to their respective controls. Among the 4 substances studied, $\beta-1,3$ glucan was found to be the most effective immunomodulator, followed by levamisole, lactoferrin and vitamin $\mathrm{C}$ in Asian catfish. Therefore, the results support the introduction of these substances into the diet of fish grown in farms under immunosuppressive/stressful conditions in order to enhance protection against infection and offer economic benefits.
\end{abstract}

KEY WORDS: Immunomodulators - Cyclophosphamide - Specific immunity · Disease resistance · Clarias batrachus $\cdot$ Aeromonas hydrophila

Resale or republication not permitted without written consent of the publisher

\section{INTRODUCTION}

Intensive fish culture systems create a highly stressful environment for fish, which, in turn, suppresses the immune response. An aquatic environment often constitutes a terminal sink for many pollutants and toxic immunosuppressive drugs (such as from waste originating from medical establishments) that further elevate the stressful environment. The Asian catfish Clarias batrachus is a high-priced, highly nutritive, air- breathing, tropical food fish that is raised in mass culture. The Asian catfish, by virtue of its preferential habitat in the bottom zones of swampy water, where the bacterial population may be 10 to 20 times higher than in the water column (Lewis \& Bender 1961), is often at a high risk of exposure to these kinds of immunosuppressive agents, and thus is prone to infection.

Cyclophosphamide (CYP), a cytotoxic drug and a well-known immunosuppressant in endotherms, has been used in the present study as an artificial immuno- 
suppressant in Clarias batrachus. Previous studies have shown that low levels of CYP exposure in fish, though not lethal, cause marked immunosuppression (Chen et al. 1983, Kitao \& Yoshida 1986, Kumari \& Sahoo 2005a).

Aeromonas hydrophila, a ubiquitous bacterium responsible for stress-associated pathogenicity in warmwater fish (e.g. carp, catfish and salmonids) is associated with small surface lesions, local haemorrhaging and septicaemia (Yin et al. 1996). Different vaccination strategies have been employed with different bacterin preparations against A. hydrophila, inducing protection against aeromoniasis in carp and catfish species with varying degrees of success (Yin et al. 1996, Nayak et al. 2004). No commercial vaccine against $A$. hydrophila is currently available for Asian catfish farming.

Recent studies of the fish immune system indicate that immunostimulants can activate fish immune functions, even in the immunosuppressive states caused by many forms of toxins or stress situations, and therefore reverse the deleterious effects mediated by stress (Anderson 1992, Sakai 1999, Sahoo \& Mukherjee 2002, 2003, Ortuno et al. 2003). Rainbow trout injected with CYP or hydrocortisone showed suppressed phagocytic activity of leucocytes, which was subsequently reversed by injection of FK-565 (Kitao \& Yoshida 1986). In aflatoxininduced immunocompromised rohu Labeo rohita, $\beta-1,3$ glucan, levamisole and vitamins $\mathrm{C}$ and $\mathrm{E}$ raised the degree of protection against Aeromonas hydrophila and Edwardsiella tarda, as well as the non-specific and specific immunity levels (Sahoo \& Mukherjee 2001a,b, $2002,2003)$. Rohu that were injected with hydrocortisone exhibited reduced innate immunity and reduced specific antibodies against E. tarda; after injection with chitosan, these fish showed an increase in immune activity (Saho \& Mukherjee 1999). Similar attempts have been made to counteract natural environmental pollutants, viz. organophosphorus and organochlorine pesticides, through the administration of chitosan, Finnstim, dimerised lysozyme and vitamin C in fish (Siwicki 1989, Dunier et al. 1995).

Previous findings showed that administration of dietary bovine lactoferrin, glucan, levamisole and vitamin $\mathrm{C}$ increased resistance to different bacterial diseases caused by Aeromonas sp., Vibrio sp., Edwardsiella sp., Yersinia ruckeri and Ichthyophthirius multifiliis in different freshwater and marine fish species (as reviewed by Mulero et al. 1998b, Sakai 1999, Sahoo \& Mukherjee 2001a,b, 2002, 2003, Kumari et al. 2003, Kumari \& Sahoo 2005b).

The present study evaluated the comparative influence of the dietary administration of the 4 proven fish immunostimulants in healthy and CYP-induced immunocompromised Asian catfish by screening spe- cific immunity against Aeromonas hydrophila bacterin and disease resistance after challenge with a virulent strain of A. hydrophila.

\section{MATERIALS AND METHODS}

Experimental animals. Fish $(32.88 \pm 1.50 \mathrm{~g})$ were obtained from a farm of the Central Institute of Freshwater Aquaculture (CIFA), Kausalyaganga, Bhubaneswar, India. Fish were acclimatised in circular cemented tanks of $700 \mathrm{l}$ capacity for $15 \mathrm{~d}$ and maintained in these tanks in dechlorinated water at 29 to $31^{\circ} \mathrm{C}$. They were fed with a formulated diet at $3 \%$ body weight once daily at 15:00 h. About $25 \%$ of the water was removed daily, along with the waste feed and faecal materials. The basic physico-chemical water parameters were measured systematically at $1 \mathrm{wk}$ intervals to maintain their optimal level (dissolved oxygen: $5.65 \pm 0.72 \mathrm{mg}$ $\mathrm{l}^{-1}$; pH: $7.41 \pm 0.82$; nitrites: $0.015 \pm 0.009 \mathrm{mg} \mathrm{l}^{-1}$; ammonia: $0.109 \pm 0.024 \mathrm{mg} \mathrm{l}^{-1}$ ) throughout the experiment.

Immunostimulants. Well-known fish immunostimulants, viz. lactoferrin (Morinaga Milk Industry), yeast $\beta-1,3$ glucan (Sigma), levamisole hydrochloride (Sigma) and vitamin C (CRNA, Roche), were used in the present experiment. The basal or control feed mix contains: fish meal, 35\%; groundnut cake, $25 \%$; soybean meal, $20 \%$; rice bran, $12 \%$; wheat flour, $5 \%$; carboxy methyl cellulose, $1 \%$; and vitamin-mineral mix, $2 \%$ (as per Kumari \& Sahoo 2005b). Based on our previous studies (Kumari et al. 2003, Kumari \& Sahoo 2005b, pers. obs.), immunostimulants were added individually to the control diet. The doses and durations of feeding for immunostimulants are as follows: lactoferrin, $100 \mathrm{mg} \mathrm{kg}^{-1}$ feed for $7 \mathrm{~d} ; \beta-1,3$ glucan, $0.1 \%$ in feed for $7 \mathrm{~d}$; levamisole, $50 \mathrm{mg} \mathrm{kg}^{-1}$ feed for $10 \mathrm{~d}$; and vitamin C, $500 \mathrm{mg} \mathrm{kg}^{-1}$ feed for $30 \mathrm{~d}$. The immunostimulant-supplemented pelleted feeds were stored at $-20^{\circ} \mathrm{C}$ after air drying, except for vitamin $\mathrm{C}$, which was prepared weekly and stored at $-20^{\circ} \mathrm{C}$.

Experimental design. Two parallel experiments were conducted, one for the measurement of specific immunity followed by challenge to vaccinated groups and the other for the non-vaccinated challenge study. Each experiment was carried out in 5 groups (Groups A, B, C, D and E for the vaccination trial and Groups a, $\mathrm{b}, \mathrm{c}, \mathrm{d}$ and e for the non-vaccination trial). In addition a control group ( $F$, with or without phosphate-buffered saline [PBS] injection) was taken, which was considered common for both vaccinated and non-vaccinated experiments. A total of 230 fish each for the vaccinated and non-vaccinated challenge studies were randomly divided into 5 groups with 42 fish in each group plus Group F containing only 20 fish. Based on healthy and 


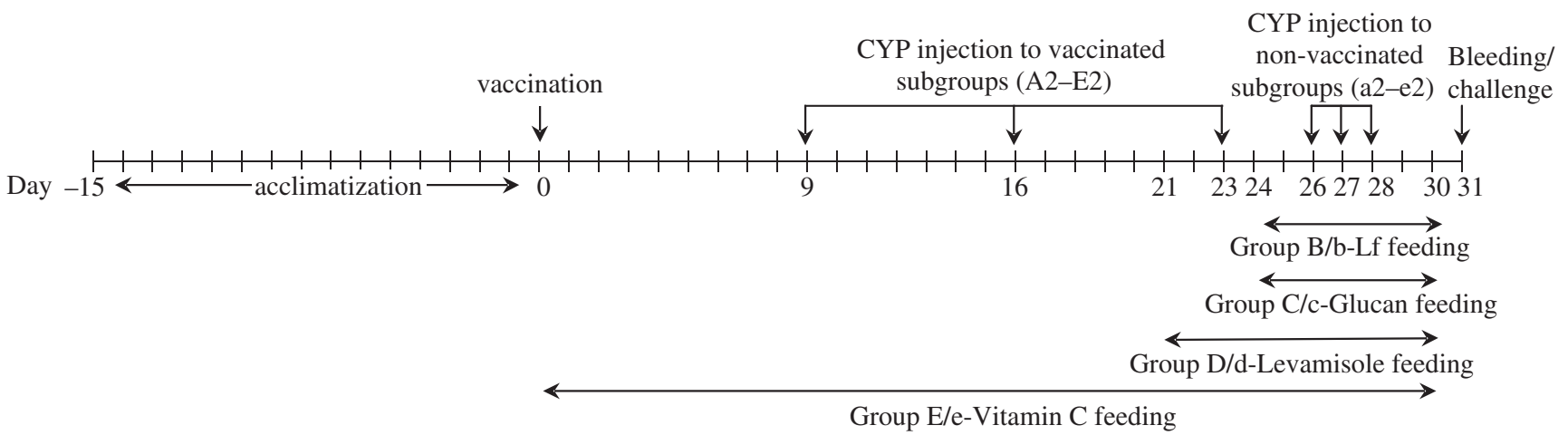

Fig. 1. Schematic diagram of the experimental design (A1-E1: vaccinated healthy; A2-E2: vaccinated CYP [cyclophosphamide]treated; a1-e1: non-vaccinated healthy; a2-e2: non-vaccinated CYP-treated)

immunocompromised fish, each group was subdivided into 2 subgroups (1 and 2) containing 7 fish in triplicate reared in individual tanks. Subgroup 1 denotes healthy fish, and Subgroup 2 denotes immunocompromised (CYP-treated) fish, except for Subgroup F1 that received only the PBS injection and F2 as the PBS negative control. Group B and C fish were fed with lactoferrin and glucan, respectively, from Days 24 to 30 of the experiment; Group D fish were provided with a levamisole-based diet from Day 21 to 30; and Group E fish received vitamin $\mathrm{C}$-mixed feed throughout the $30 \mathrm{~d}$ of the experimental period (Fig. 1).

In Expt 1, fish of Groups A, B, C, D and E (both Subgroups 1 and 2) were vaccinated on Day 1 and fish of Subgroup F1 were injected with PBS. Fish of Subgroups A2, B2, C2, D2 and E2 were injected with CYP intraperitoneally (i.p.) at a dose of $200 \mathrm{mg} \mathrm{kg}^{-1}$ on Days 9, 16 and 23 after vaccination and were bled and challenged with Aeromonas hydrophila on Day 31. Expt 2 was run in non-vaccinated fish with similar groupings and subgroupings. Subgroups a1 to e1 were treated as non-vaccinated healthy animals, whereas fish of Subgroups a2 to e2 were injected with CYP i.p. on Days 26, 27 and 28 in a previously described manner (Kumari \& Sahoo 2005a). On Day 31, fish were challenged with live A. hydrophila as described earlier (Kumari \& Sahoo 2005a). A gap of $7 \mathrm{~d}$ between CYP injections in vaccinated fish was scheduled to ensure the optimum suppression observed within an earlier $30 \mathrm{~d}$ trial (Kumari \& Sahoo 2005a). The challenge was conducted within 3 to $8 \mathrm{~d}$ of the last CYP injection according to previous observations of earlier researchers who noted a partial immune recovery after 4 to $8 \mathrm{~d}$ of CYP injections (Chen et al. 1983, Kumari \& Sahoo 2005a).

All fish were anaesthetised with a sedative dose of 2-phenoxyethanol (Sigma) (100 l phenoxyethanol $1^{-1}$ water) (Kumari et al. 2003) prior to any injection or blood collection from the caudal vein, to minimise the possible stress induced during handling.
Bacterin preparation and vaccination. Formalinkilled Aeromonas hydrophila bacterin was prepared according to Kumari et al. (2003), and $0.1 \mathrm{ml}$ of suspension was injected i.p. into each fish on Day 1 of the experiment, except in the negative control group $(F)$. Ten fish of Subgroup F1 were injected i.p. with $0.1 \mathrm{ml}$ of PBS instead of bacterin. Then, 42 fish of each group were utilised for the vaccinated challenge experiment, 21 of which were used directly for the challenge study and the remaining 21 fish were used to measure circulating antibody titres. All assays were performed in triplicate, and the mean $( \pm \mathrm{SE})$ for each subgroup was calculated.

Agglutinating antibody assay. On Day 31, 3 fish of each replicate were bled from each subgroup, and serum samples were collected and stored at $-20^{\circ} \mathrm{C}$ until further analysis. Antibody titres against the homologous isolate of Aeromonas hydrophila were determined as described by Kumari et al. (2003). Twofold serial dilutions of serum samples were incubated with washed, formalin-killed $A$. hydrophila $\left(10^{9}\right.$ cells $\mathrm{ml}^{-1}$ ) at room temperature overnight. The antibody titres were expressed as the reciprocal of the highest serum dilution giving positive agglutination.

Disease resistance study. Vaccinated and nonvaccinated challenge experiments in Clarias batrachus were simultaneously conducted to study the protection level of healthy or immunocompromised fish fed with various immunostimulants against the virulent Aeromonas hydrophila challenge. All the fish, in triplicate (7 fish $\operatorname{tank}^{-1}$ ), from each subgroup, i.e. healthy/ immunocompromised (CYP-treated) and fed with/ without immunostimulants, were injected i.p. with $0.1 \mathrm{ml}$ of $10^{6}$ live cells of $A$. hydrophila per fish. The percent survival was recorded up to $10 \mathrm{~d}$, based on our earlier observations that mortality reached a plateau after $1 \mathrm{wk}$. The cause of mortality was confirmed by reisolating the organism from the kidney of $10 \%$ of the dead catfish as described earlier (Kumari et al. 2003). 
Statistical analysis. Percent survival data were normalised using arcsine transformation before analysis. Data were analysed using 1-way or 2-way ANOVA. Means were compared using Duncan's multiple range tests to determine the significant difference at the $5 \%$ $(\mathrm{p}<0.05)$ level.

\section{RESULTS}

\section{Antibody titre}

The specific humoral immune response measured by bacterial agglutination titre against formalin-killed Aeromonas hydrophila is shown in Fig. 2. The fish of Subgroup C1 showed the highest antibody titre against A. hydrophila among all the subgroups, followed by Subgroup D1. In immunocompromised fish, CYP injections (i.p.) on 3 occasions at weekly intervals significantly $(p<0.05)$ suppressed the antibody titre of the A2 subgroup fish compared to the healthy control subgroup (A1). The antibody titre was significantly enhanced in all the immunostimulant-fed groups compared to their respective controls. Remarkably, the suppressed antibody titre observed in Subgroup A2

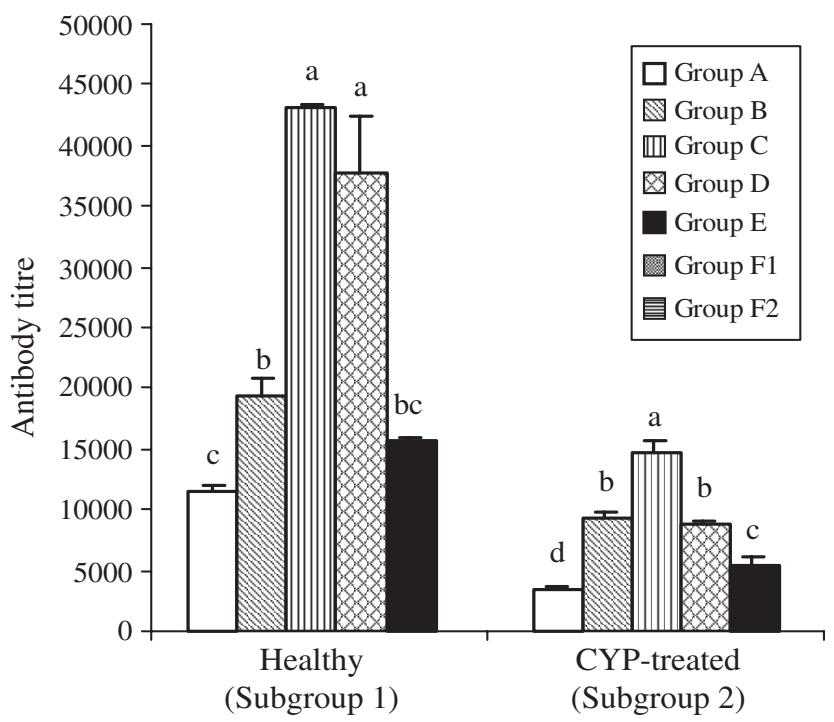

Fig. 2. Clarias batrachus. Serum antibody titre after vaccination with formalin-killed Aeromonas hydrophila in healthy and immunocompromised (CYP-treated) catfish with or without exposure to various immunostimulants. Non-vaccinated PBS positive and PBS negative control subgroups (F1 and F2, respectively) were seronegative for $A$. hydrophila (and hence not shown). Data are means $( \pm \mathrm{SE})$. Statistical differences between controls and different immunostimulated groups indicated by letters $(\mathrm{a}, \mathrm{b}, \mathrm{c}, \mathrm{d})$ over the bars. Group A: vaccinated control; Group B: lactoferrin fed; Group C: glucan fed; Group D: levamisole fed; Group E: vitamin C fed

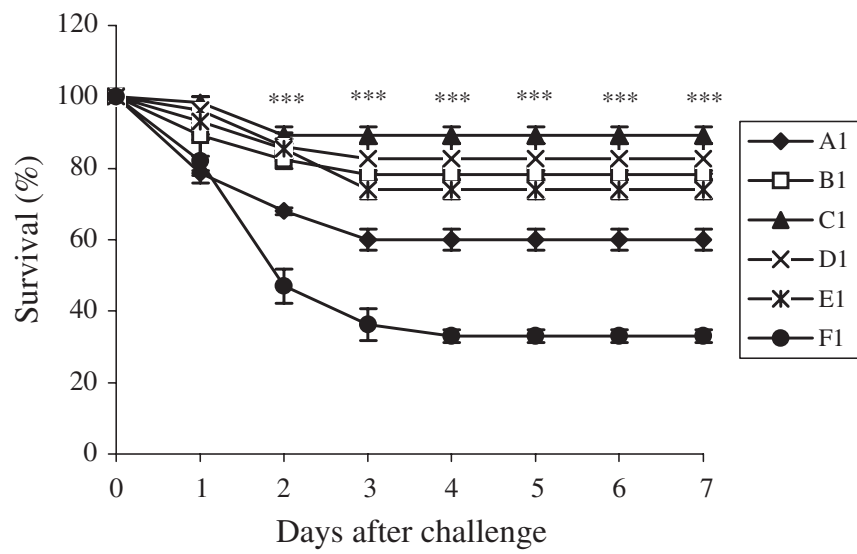

Fig. 3. Clarias batrachus. Effect of immunostimulants on the percent survival of healthy vaccinated catfish challenged with Aeromonas hydrophila. Data are means $( \pm \mathrm{SE}) .{ }^{* * *} \mathrm{p}<0.001$. Group A1: vaccinated control; Group B1: lactoferrin-fed; Group C1: glucan-fed; Group D1: levamisole-fed; Group E1: vitamin C-fed; Group F1: PBS-treated

was restored to a level near or above normal (A1 level) by using each of the immunostimulants (Fig. 2). The Subgroups F1 and F2 were found to be seronegative (considered when agglutination titre was $<4$ ) for A. hydrophila antibodies.

\section{Disease resistance}

The cumulative percent survival after $10 \mathrm{~d}$ of challenge with Aeromonas hydrophila in both vaccinated and non-vaccinated fish is presented in Tables $1 \& 2$, and survival over a $7 \mathrm{~d}$ period is depicted in Figs. 3 to 6.

As shown in Fig. 3, the percent survival became steady after 2 and $4 \mathrm{~d}$ of Aeromonas hydrophila challenge in Subgroups C1 and F1, respectively, and after $3 \mathrm{~d}$ in Subgroups A1, B1, D1 and E1. The minimum percent survival after 3 and $4 \mathrm{~d}$ of challenge was found to be $33 \%$ in Subgroup F1 and $60 \%$ in Subgroup A1, and the highest survival rate $(89.33 \%)$ was observed in Subgroup C1, followed by Subgroups D1 (82.67\%), B1 $(78.17 \%)$ and E1 $(74.00 \%)$. At all time points, except $1 \mathrm{~d}$ after challenge, all the immunostimulant-fed vaccinated healthy subgroups showed a significantly higher percent survival compared to A1 and F1 subgroups.

On the other hand, in CYP-exposed vaccinated subgroups survival of 28.33 and $33 \%$ were recorded in Subgroups A2 and F2 after 4 d of challenge, respectively. Subgroup C2 showed a significantly higher percent survival after just $2 \mathrm{~d}$ of challenge. Further, a significantly higher percent survival was obtained in all the immunostimulant-fed, CYP-treated subgroups 3 d post-challenge compared to Control Subgroup A2 (Fig. 4). 


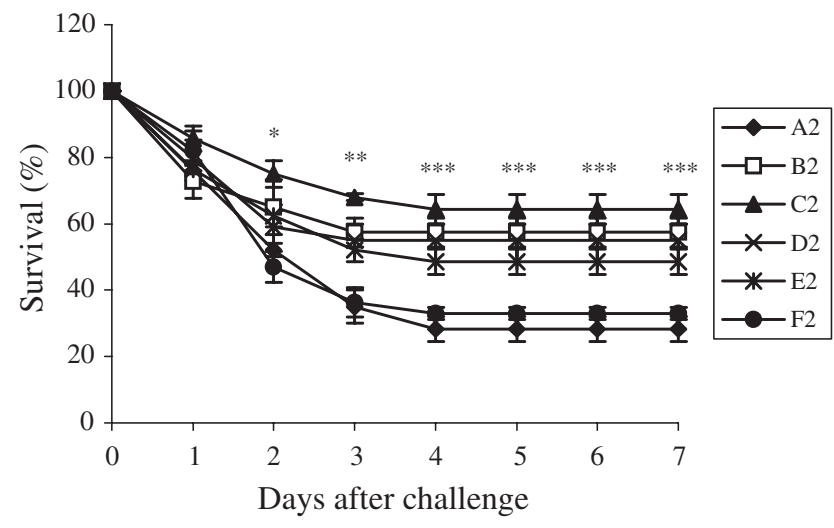

Fig. 4. Clarias batrachus. Effect of immunostimulants on the percent survival of cyclophosphamide-exposed vaccinated catfish challenged with Aeromonas hydrophila. Data are means $( \pm \mathrm{SE}) .{ }^{*} \mathrm{p}<0.05,{ }^{* *} \mathrm{p}<0.01{ }^{* * *} \mathrm{p}<0.001$. Group A2: vaccinated control; Group B2: lactoferrin-fed; Group C2: glucan-fed; Group D2: levamisole-fed; Group E2: vitamin Cfed; Group F2: healthy control

The cumulative percent survival in healthy Subgroups A1, B1, C1, D1 and E1 were higher than in their respective immunocompromised subgroups $10 \mathrm{~d}$ postchallenge. The percentage survival was restored back to control subgroup levels in the immunostimulant-fed, CYP-treated subgroups (B2, C2, D2 and E2) (Table 1). The highest mortality rate was obtained in Group F fish irrespective of PBS exposure.

The mortality rate became steady after $2 \mathrm{~d}$ of challenge in Subgroup c1, after 3 d in Subgroups a1, d1 and 1 and after $4 \mathrm{~d}$ in Subgroup b1 in non-vaccinated, challenged groups (Fig. 5). Of the healthy nonvaccinated subgroup the lowest survival (35\%) $3 \mathrm{~d}$ after challenge was observed in Subgroup a1. Furthermore, $1 \mathrm{~d}$ post-challenge, only Subgroups b1 and d1 showed a significantly higher percent survival compared to Subgroup a1.

On the other hand, in non-vaccinated, CYP-exposed subgroups, only $5 \%$ survival was obtained in Subgroup a2 six days post-challenge, compared to 45,51 , 37 and $40 \%$ for Subgroups b2, c2, d2 and e2, respec-

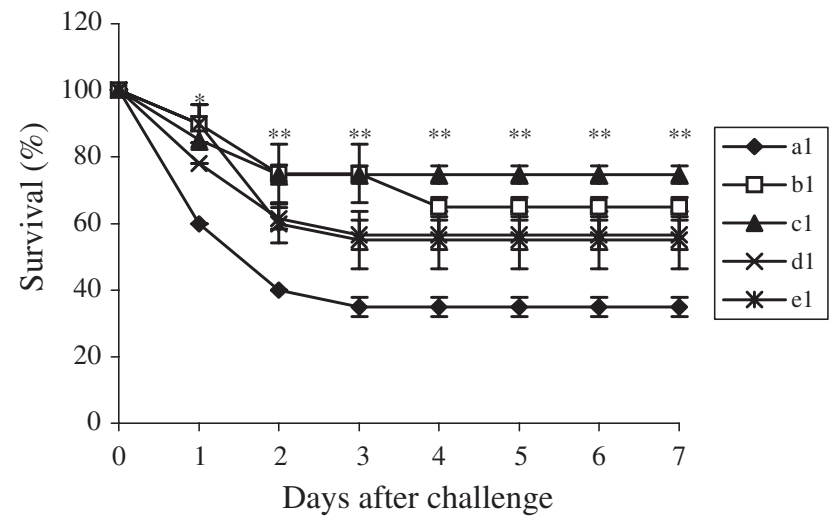

Fig. 5. Clarias batrachus. Effect of immunostimulants on the percent survival of healthy non-vaccinated catfish challenged with Aeromonas hydrophila. Data are means $( \pm \mathrm{SE}) .{ }^{*} \mathrm{p}<0.05$ and ${ }^{* *} \mathrm{p}<0.01$. Group a1: non-vaccinated control; Group b1: lactoferrin-fed; Group c1: glucan-fed; Group d1: levamisolefed; Group e1: vitamin C-fed

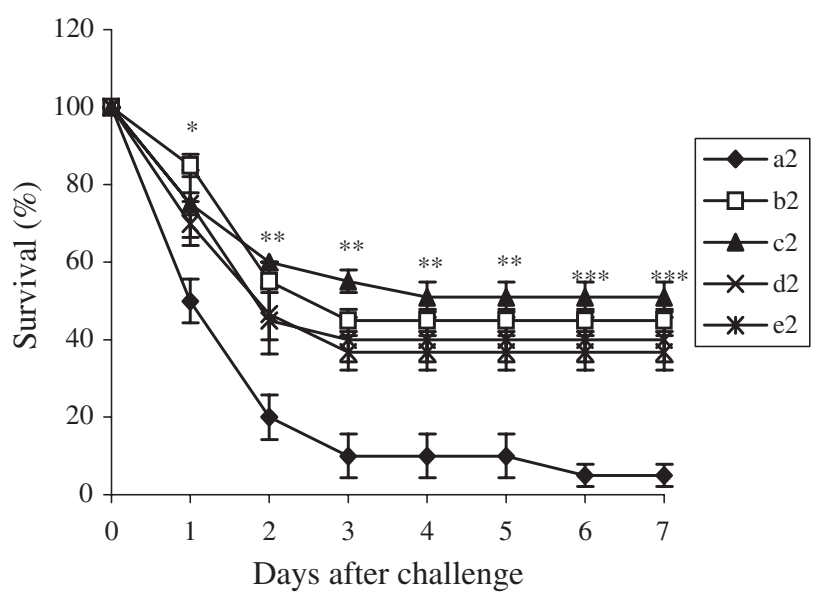

Fig. 6. Clarias batrachus. Effect of immunostimulants on the percent survival of cyclophosphamide-exposed non-vaccinated catfish challenged with Aeromonas hydrophila. Data are means $\left( \pm\right.$ SE). ${ }^{*} p<0.05,{ }^{* *} p<0.01$ and ${ }^{* * *} p<0.001$ Group a2: non-vaccinated control; Group b2: lactoferrin-fed; Group c2: glucan-fed; Group d2: levamisole-fed; Group e2: vitamin C-fed

Table 1. Clarias batrachus. Cumulative percent survival of vaccinated catfish after exposure to cyclophosphamide (CYP) and/or immunostimulants and control groups after challenge with Aeromonas hydrophila. Data represent means $( \pm \mathrm{SE})$. Means bearing different superscripts $(\mathrm{l}, \mathrm{m}, \mathrm{n})$ row-wise are significantly different $(\mathrm{p}<0.05)$. PBS: phosphate-buffered saline. Differences between healthy and CYP-treated fish were significant in all cases. For fish groups see Figs. 3 \& 4

\begin{tabular}{|lccccccc|}
\hline & Control & Lactoferrin & Glucan & Levamisole & Vitamin C & $\begin{array}{c}\text { PBS-treated } \\
\left.\text { (non-vaccinated, } \mathrm{F}_{1}\right)\end{array}$ & $\begin{array}{c}\text { PBS-untreated } \\
\left(\text { non-vaccinated, } \mathrm{F}_{2}\right)\end{array}$ \\
\hline Healthy fish & $60.0^{\mathrm{n}}$ & $78.2^{\mathrm{m}}$ & $89.3^{\mathrm{l}}$ & $82.7^{\mathrm{I}^{\mathrm{m}}}$ & $74.0^{\mathrm{m}}$ & $33^{\mathrm{n}}$ & $35^{\mathrm{n}}$ \\
(A1, B1, C1, D1 and E1) & \pm 2.9 & \pm 1.2 & \pm 2.3 & \pm 4.3 & \pm 2.6 & \pm 1.8 & \pm 2.9 \\
CYP-treated fish & $28.3^{\mathrm{n}}$ & $57.3^{\mathrm{mm}}$ & $63.3^{\mathrm{l}}$ & $55.0^{\mathrm{lm}}$ & $48.5^{\mathrm{m}}$ & & \\
(A2, B2, C2, D2 and E2) & \pm 3.8 & \pm 4.3 & \pm 4.4 & \pm 5.0 & \pm 3.8 & \\
\hline
\end{tabular}


Table 2. Clarias batrachus. Cumulative percent survival of non-vaccinated catfish after exposure to cyclophosphamide and/or immunostimulants and control groups after challenge with Aeromonas hydrophila. Data represent means $( \pm$ SE). Means bearing different superscripts $(1, m, n)$ row-wise and subscripts $(x, y)$ column-wise are significantly different $(p<0.05)$. PBS: phosphatebuffered saline. For fish groups see Figs. $5 \& 6$

\begin{tabular}{|lccccccc|}
\hline & Control & Lactoferrin & Glucan & Levamisole & Vitamin C & $\begin{array}{c}\text { PBS-treated } \\
\left.\text { (non-vaccinated, } \mathrm{F}_{1}\right)\end{array}$ & $\begin{array}{c}\text { PBS-untreated } \\
\left(\text { non-vaccinated, } \mathrm{F}_{2}\right)\end{array}$ \\
\hline Healthy fish & $35^{\mathrm{n}}$ & $65.0^{\mathrm{lm}}$ & $74.7^{\mathrm{l}}$ & $56.7^{\mathrm{m}}$ & $55.0^{\mathrm{m}}$ & $33^{\mathrm{n}}$ & $35^{\mathrm{n}}$ \\
(a1, b1, c1, d1 and e1) & $\pm 2.9_{\mathrm{x}}$ & $\pm 2.9_{\mathrm{x}}$ & $\pm 2.6_{\mathrm{x}}$ & $\pm 4.4_{\mathrm{x}}$ & $\pm 8.7_{\mathrm{x}}$ & \pm 1.8 & \pm 2.9 \\
CYP-treated fish & $5.0^{\mathrm{n}}$ & $45.0^{\mathrm{lm}}$ & $51.0^{\mathrm{l}}$ & $36.7^{\mathrm{m}}$ & $40.0^{\mathrm{m}}$ & & \\
(a2, b2, c2, d2 and e2) & $\pm 2.9_{\mathrm{y}}$ & $\pm 2.9_{\mathrm{y}}$ & $\pm 3.8_{\mathrm{y}}$ & $\pm 4.4_{\mathrm{y}}$ & $\pm 5.8_{\mathrm{x}}$ & \\
\hline
\end{tabular}

tively (Fig. 6). Similar to Subgroup c1, Subgroup c2 showed the highest percent survival. Furthermore, all the immunostimulant-fed subgroups showed a significant increase in percent survival starting from $1 \mathrm{~d}$ after challenge compared to Control Subgroup a2, thus clearly showing the immunoreversal effect of all 4 immunostimulants.

Similar to the vaccinated fish, feeding of glucan to non-vaccinated healthy and immunocompromised catfish enhanced the percentage survival by 74.7 and $51 \%$, respectively, compared to other substances (Table 2). However, all 4 substances increased the percent survival notably in both healthy and immunocompromised fish compared to their respective controls, and the percent survival was restored back to or above the normal levels of Subgroups b2, c2, d2 and e2 (Table 2). Besides, the percent survival in vaccinated fish was observed to be marginally higher than that of nonvaccinated fish.

\section{DISCUSSION}

The current research is directed towards an alternative approach-the use of immunostimulants along with bacterin to boost or stimulate the specific immune response as well as the protection against aeromoniasis, apparently through a more complete exploitation of the host's immune system. Few studies have focussed on the oral administration of different immunostimulants and their comparative efficacies in vaccinated healthy as well as immunosuppressed fish to increase disease resistance. Results are especially scarce with respect to levamisole, glucan and vitamin $\mathrm{C}$, and there are no available data concerning the use of lactoferrin in immunosuppressed fish. Therefore, the current study, the first of its type for Clarias batrachus, was conducted in order to evaluate the protective role of these immunostimulants against the immunosuppressive response induced by CYP in both vaccinated and non-vaccinated fish.
Immunostimulants used along with fish bacterin, viz. FCA, FIA, BCG, FK-565, saponin, Quit A, levamisole and glucans, could enhance immune responses and protection to some degree (Nikl et al. 1991, Anderson \& Jeney 1992). Research on immunostimulants added directly to bacterin and their stability or potency is poorly defined. However, dietary intake of immunostimulants in fish has definite advantages, and is a useful method of exposure in large-scale fish culture (Siwicki et al. 1994).

In the present study, the lowered antibody response against Aeromonas hydrophila observed in the CYPtreated control subgroup (A2) compared to the healthy control subgroup (A1) was restored to normal levels by feeding immunostimulants. Indeed, the feeding of each immunostimulant to healthy and immunocompromised groups significantly raised the antibody titres compared to the respective controls. Among the 4 substances used, the antibody titre of the dietary glucan group showed a significantly higher level, thus clearly exhibiting its efficacy in comparison to the other 3 substances. This finding is in accordance with the observations made by Nikl et al. (1991), who evaluated 7 immunostimulants for their protective effects against A. salmonicida infection in coho salmon. Similarly, Sahoo \& Mukherjee (2002) evaluated 4 well-known immunostimulants in healthy and immunocompromised (aflatoxin-treated) rohu regarding their protection against Edwardsiella tarda infection; they observed $\beta-1,3$ glucan to be the most potent immunostimulant. The effects of lactoferrin, levamisole, glucan and vitamin $\mathrm{C}$ as feed additives and immunostimulants in the present study point to the reversal of drugrelated immunosuppression in vaccinated and nonvaccinated fish. The significant reduction in antibody titre in Subgroup A2 compared to Subgroup A1 may be due to the fact that CYP exerts a more depressive effect on antibody production if given after antigenic stimulation. This may be due to interference of helper cell activity (Santos 1967). According to Figueras et al. (1998), the highest activities of immunostimulants on 
immune parameters may be obtained after bacterin exposure. Therefore, all the immunostimulants were administered after bacterin exposure. The bacterial agglutination titre was not measured in nonvaccinated fish. The naive or F1 and F2 subgroups of fish used in the vaccinated trial, obtained from the same source as the non-vaccinated fish, revealed antibody titre $<4$; thus, they were considered seronegative in this study. This negligible antibody titre might be due to the presence of natural antibodies. However, the haemagglutinin level of rabbit red blood cells measured in non-vaccinated subgroups did not differ notably after exposure to various immunostimulants or to CYP (pers. obs.).

Although, aeromoniasis is still a common disease causing severe losses in many countries, effective prophylactic or control measures are still needed. It may be very difficult to tackle an Aeromonas hydrophila epizootic, particularly if fish are immunosuppressed. This study has shown that it is possible to raise the protection level under immunosuppressive conditions; this successful protection/control may provide insight for further research and applications for aquaculture.

In the disease resistance study against Aeromonas hydrophila, the cumulative percent survival observed in Subgroup a1 was similar to that seen in the vaccinated, CYP-treated immunocompromised subgroup (A2). The feeding of glucan, lactoferrin, levamisole and vitamin $\mathrm{C}$ to the vaccinated and non-vaccinated healthy fish significantly enhanced the protection level compared to Subgroup A1. Similarly, in the case of immunocompromised subgroups, all 4 immunostimulants significantly reduced mortality in CYP-treated fish compared to Subgroup a2, thus clearly exhibiting immunoreversal events and thus their effectiveness for fish farming. Evidence suggests that the degree of protection obtained in non-vaccinated fish by dietary lactoferrin could be related to the stimulation of nonspecific components of the immune system. A similar high survival rate after challenge with fish-pathogenic bacteria has been reported after glucan exposure (Anderson 1992, Sakai 1999, Sahoo \& Mukherjee 2001b, 2002, Kumari \& Sahoo pers. obs.). Evidence suggests that the glucan-like lactoferrin enhances disease resistance by stimulating a non-specific component of the immune system (Sakai 1999) or through better processing and presentation of antigen-enhancing specific defences. In the findings of the present study, enhanced antibody titre (only in vaccinated fish) and maximum protection in glucan-fed, vaccinated and non-vaccinated CYP-treated subgroups compared to the other groups were noteworthy. In addition, the survival rate and antibody titre observed in the vaccinated, levamisole-fed Subgroup D1 were similar to those in the glucan-fed Subgroup C1. This increased protection may be correlated with increased phagocytosis (Symoens \& Rosenthal 1977, Mulero et al. 1998a), cytokine expression by macrophages (Kimball et al. 1992), lymphocyte proliferation following exposure to mitogens (Woods et al. 1974) and antibody responses (Tempero et al. 1995) observed during levamisole treatment in other fish species.

The cumulative percent survival as observed in fish fed with vitamin $\mathrm{C}$ (both vaccinated and nonvaccinated) was similar to that of the lactoferrin and levamisole groups. The positive influence of vitamin $\mathrm{C}$ on disease resistance has been described earlier in many fish species (Navarre \& Halver 1989, Sahoo \& Mukherjee 2002, 2003, Lin \& Shiau 2005, Kumari \& Sahoo 2005b). Glucan was the most effective of the 4 substances in protecting fish against bacterial infection and also had the best immune reversal effect.

To conclude, the described approach evaluated the possibility of reducing the deleterious effects on the immune system of Asian catfish caused by exposure to CYP. The present findings suggest a dietary supplementation of lactoferrin, glucan, levamisole and vitamin $\mathrm{C}$ to increase protection against infectious diseases, especially under immunocompromised and stressful conditions.

Acknowledgements. The authors thank the director of the Central Institute of Freshwater Aquaculture (CIFA), Kausalyaganga, Bhubaneswar, India, and the Catfish Unit (CIFA) for providing the requisite facilities and fish, respectively, during the study.

\section{LITERATURE CITED}

Anderson DP (1992) Immunostimulants, adjuvants, and vaccine carriers in fish: applications to aquaculture. Annu Rev Fish Dis 2: 281-307

Anderson DP, Jeney G (1992) Immunostimulants added to injected Aeromonas salmonicida bacterin enhance the defense mechanisms and protection in rainbow trout (Oncorhynchus mykiss). Vet Immunol Immunopathol 34: 379-389

Chen MF, Rohovec JS, Fryer JL (1983) Effect of methotrexate, kenalog, and cyclophosphamide on the antibody response of steelheads to Aeromonas salmonicida. Trans Am Fish Soc 112:561-564

Dunier M, Siwicki AK, Verlhac V, Vergnet C, Studnicka M (1995) The immunotoxic effect of Lindane on the specific and nonspecific immune response of rainbow trout and treatment with Nitrogranulin and Vitamin C. In: Stolen JF (Ed) Modulators of immune responses. SOS Publications, Fair Haven, NJ, p 145 (abstract)

Figueras A, Santarem MM, Novoa B (1998) Influence of the sequence of administration of b-glucans and a Vibrio damsela vaccine on the immune response of turbot (Scophthalmus maximus L.). Vet Immunol Immunopathol 64:59-68

Kimball ES, Schneider CR, Fisher MC, Clark MC (1992) Levamisole causes differential cytokine expression by elicited mouse peritoneal macrophages. J Leukoc Biol 52:349-356 
Kitao T, Yoshida Y (1986) Effect of an immunopotentiator on Aeromonas salmonicida infection in rainbow trout (Salmo gairdneri). Vet Immunol Immunopathol 12:287-296

Kumari J, Sahoo PK (2005a) Effects of cyclophosphamide on the immune system and disease resistance of Asian catfish, Clarias batrachus. Fish Shellfish Immunol 19:307-316

Kumari J, Sahoo PK (2005b) High dietary vitamin C affects growth, non-specific immune responses and disease resistance in Asian catfish, Clarias batrachus. Mol Cell Biochem 280:25-33

Kumari J, Swain T, Sahoo PK (2003) Dietary bovine lactoferrin induces changes in immunity level and disease resistance in Asian catfish Clarias batrachus. Vet Immunol Immunopathol 94:1-9

Lewis WM, Bender M (1961) Free-living ability of a warmwater fish pathogen of the genus Aeromonas hydrophila - standardization of dose and duration for oral vaccination of carps. Fish Shellfish Immunol 9:519-528

Lin MF, Shiau SY (2005) Dietary L-ascorbic acid affects growth, nonspecific immune responses and disease resistance in juvenile grouper, Epinephelus malabaricus. Aquaculture 244:215-221

Mulero V, Esteban MA, Meseguer J (1998a) Effects of in vitro addition of exogenous vitamins $\mathrm{C}$ and $\mathrm{E}$ on gilthead seabream (Sparus aurata L.) phagocytes. Vet Immunol Immunopathol 66:185-199

Mulero V, Esteban MA, Munoz J, Meseguer J (1998b) Dietary intake of levamisole enhances the immune response and disease resistance of the marine teleost gilthead seabream (Sparus aurata). Fish Shellfish Immunol 8:49-62

Navarre O, Halver J (1989) Disease resistance and humoral antibody production in rainbow trout fed high levels of vitamin C. Aquaculture 79:207-221

Nayak DK, Asha A, Shankar KM, Mohan CV (2004) Evaluation of biofilm of Aeromonas hydrophila for oral vaccination of Clarias batrachus-a carnivore model. Fish Shellfish Immunol 16:613-619

Nikl L, Albright LJ, Evelyn TPT (1991) Influence of seven immunostimulants on the immune response of coho salmon to Aeromonas salmonicida. Dis Aquat Org 12:7-12

Ortuno J, Esteban MA, Meseguer J (2003) The effect of dietary intake of vitamins $\mathrm{C}$ and $\mathrm{E}$ on the stress response of gilthead seabream (Sparus aurata L.). Fish Shellfish Immunol 14:145-146

Editorial responsibility: Donald L. Evans, Athens, Georgia, USA
Sahoo PK, Mukherjee SC (1999) Influence of the immunostimulants, chitosan on immune responses of healthy and cortisol-treated rohu (Labeo rohita). J Aquacult Trop 14: 209-215

Sahoo PK, Mukherjee SC (2001a) Dietary intake of levamisole improves non-specific immunity and disease resistance of healthy and aflatoxin-induced immunocompromised rohu, Labeo rohita. J Appl Aquacult 11:15-25

Sahoo PK, Mukherjee SC (2001b) Effect of dietary $\beta-1,3$ glucan on immune responses and disease resistance of healthy and aflatoxin $\mathrm{B}_{1}$-induced immunocompromised rohu (Labeo rohita Hamilton). Fish Shellfish Immunol 11:683-695

Sahoo PK, Mukherjee SC (2002) Effect of dietary immunomodulation upon Edwardsiella tarda vaccination in healthy and immunocompromised Indian major carp (Labeo rohita). Fish Shellfish Immunol 12:1-16

Sahoo PK, Mukherjee SC (2003) Immunomodulation by dietary vitamin $\mathrm{C}$ in healthy and aflatoxin $\mathrm{B}_{1}$-induced immunocompromised rohu (Labeo rohita). Comp Immunol Microbiol Infect Dis 26:65-76

Sakai M (1999) Current research status of fish immunostimulants. Aquaculture 172:63-92

Santos GW (1967) Immunosuppressive drugs. I. Fed Proc 26 : 907-913

Siwicki AK (1989) Immunostimulating influence of levamisole on nonspecific immunity in carp (Cyprinus carpio). Dev Comp Immunol 13:87-89

Siwicki AK, Anderson DP, Rumsey GL (1994) Dietary intake of immunostimulants by rainbow trout affects non-specific immunity and protection against furunculosis. Vet Immunol Immunopathol 41:125-139

Symoens J, Rosenthal M (1977) Levamisole in the modulation of the immune response. The current experimental and clinical state. J Reticuloendothel Soc 21:175-221

Tempero MA, Haga Y, Sivinski C, Birt D, Klassen L, Thiele G (1995) Immunological effects of levamisole in mice and humans: evidence for augmented antibody response without modulation of cellular cytotoxicity. J Immunother 17:47-57

Woods WA, Siegel MJ, Chirigos MA (1974) In vitro stimulation of spleen cell cultures by poli Y: poly C and levamisole. Cell Immunol 14: 327-331

Yin Z, Lam TJ, Sin YM (1996) The role of specific antiserum of catfish, Clarias gariepinus, as a defence against Aeromonas hydrophila. Fish Shellfish Immunol 6:57-69

Submitted: May 16, 2005; Accepted: January 1, 2006

Proofs received from author(s): May 19, 2006 